\title{
Micro-Diversity Analysis of Error Probability and Channel Capacity over Hoyt-Gamma Fading
}

\author{
Sandeep KUMAR ${ }^{1}$, Sanjay Kumar SONI², Priyanka JAIN ${ }^{1}$ \\ ${ }^{1}$ Dept. of Electronics and Communication, Delhi Technological University, Delhi, India \\ ${ }^{2}$ Dept. of Electronics and Communication, G.B. Pant Engineering College, Pauri, UK, India \\ sann.kaushik@gmail.com,sksoni98fece@gbpec.ac.in,priyajain2000@rediffmail.com
}

Submitted January 24, 2017 / Accepted May 1, 2017

\begin{abstract}
In wireless communication system, various parameters such as average symbol/bit error probability (ASEP/ABEP), outage probability and average channel capacity, etc. are studied for its performance analysis. In this paper, the performance of L-Hoyt/Gamma (HG) composite fading channel with Maximum Ratio Combining (MRC) employing micro-diversity is analyzed. Closed-form expressions for distribution function, moments, outage probability and channel capacity are derived in terms of hypergeometric functions. Further, the expressions of $A S E P / A B E P$ for all formats of coherent and non-coherent modulation techniques involving $Q$-functions and Marcum $Q$-function are derived over the composite fading channel. Monte-Carlo simulations are performed to validate our analytical results. The analytical results produced here can be useful in several wireless applications where multipath and shadowing are characterized by Hoyt/Gamma distribution.
\end{abstract}

\section{Keywords}

Wireless communication system, shadowing, composite fading, diversity combining, MRC, channel capacity

\section{Introduction}

Composite fading environments are frequently encountered in mobile radio propagation in which multipath fading is superimposed on shadowing [1]. Multipath fading is captured by several distributions like Rayleigh, Weibull, Nakagami-m and Hoyt. Hoyt distribution is commonly used to model the short-term fading characteristics of wireless communication systems with more severe fading conditions [2], [3]. Closed-form expressions for probability distribution function (PDF), moment generating function (MGF) and high-order moments of Hoyt distribution were obtained in [1], [4], and the expression for cumulative distribution function (CDF) was presented in [5]. The closed-form analysis for the outage probability of Hoyt fading channel under Rayleigh interference and Rayleigh fading channel under mixed Rayleigh and Hoyt interference were evaluated in [6], [7].
In shadowed fading, large-scale signal variation can be described in the form of two distributions [2]. These statistical models are lognormal distribution and Gamma distribution. Shadowing has usually been modeled using lognormal distribution. However, using the lognormal PDF often leads to analytical complexities, because it is not possible to write the closed-form mathematical expressions for performance parameters of the system [2]. An alternate approach was adopted in [8], [9], where shadowing is modeled by cascading short-term fading, while Holtzman approximation was used in [10] to find the closed-form expressions of the Weibull-lognormal composite fading channel. All the above approaches have their own limitations in the sense that they do not give accurate results over the whole range of the composite fading parameters. Gamma distribution is the most acceptable approximation of lognormal distribution and is most widely used in literature to model shadowing effects [11]. The performance of several composite distributions like K [11], generalized-K [12] and the Weibull-Gamma [13] using gamma function have been analyzed in literature. Specifically, in [14] a general model of a class of composite fading such as $\eta-\mu /$ Gamma is carried out, where the result, as a special case, reduced to Hoyt-Gamma case is derived.

Short-term fading is mitigated through micro-diversity while macro-diversity approach is used to overcome the effect of long-term fading. Hinging on the fact that selection combining (SC) diversity enjoys the lowest implementation complexity, the performance of macro-diversity and micro-diversity systems using SC were studied in [15], [16]. MRC gives the best performance of all the diversity techniques, and in [17], the performance of MRC receiver over Hoyt fading was studied. However, to the best of author's knowledge, performance analysis of wireless communication system with micro-diversity, operating over Gamma shadowed Hoyt multipath fading channels is not reported in open literature.

Motivated by this fact, we have derived closed-form solution of L-HG composite fading, where Hoyt represents multipath fading and Gamma captures the shadowing effect. Performance metrics such as the amount of fading (AF), channel capacity and outage probability in terms of output signal-to-noise ratio (SNR) have been derived. The 
symbol error rates (SER) of coherent schemes are evaluated in the closed-form, and a single generalized result, encompassing all linear modulation formats is presented. Further, closed-form expressions of error probability of non-coherent techniques involving Marcum Q-function are derived and compared with the coherent schemes.

The rest of the paper is organized as follows. In Sec. 2, the $L-H G$ distribution is derived, and the performance parameters such as AF and outage probability, have been calculated. Channel Capacity has been discussed in Sec. 3. In Sec. 4, ASEP/ABEP of coherent modulation schemes are derived, and in Sec. 5 mathematical expressions for non-coherent schemes are evaluated. In Sec. 6, we present the discussion on the closed-form results obtained in the preceding sections. Finally, Section 7 concludes the work.

\section{HG Statistics}

PDF of received SNR per symbol of conditional Hoyt fading channel is given by [1]

$$
\begin{aligned}
& p_{\gamma_{l}}\left(\gamma_{l} / w\right)= \\
& \frac{1+q^{2}}{2 q w} \exp \left[-\left(\frac{\left(1+q^{2}\right)^{2} \gamma_{l}}{4 q^{2} w}\right)\right] \mathrm{I}_{0}\left(\frac{\left(1-q^{4}\right) \gamma_{l}}{4 q^{2} w}\right), \gamma_{l} \geq 0
\end{aligned}
$$

where $\gamma_{l}$ is the instantaneous received SNR of the $l^{\text {th }}$ branch and $w$ is the average received SNR of the conditional Hoyt distribution. $q$ is the Hoyt fading parameter, having the value from 0 to 1 and $\mathrm{I}_{0}(\cdot)$ is the modified Bessel of the first type and zeroth order. Short-term fading is mitigated through micro-diversity approaches using multiple antennas at the receiver [2]. When MRC diversity is used, the overall SNR at the output of the receiver is the sum of SNR of all the individual branches multiplied by their proportional weights. Instantaneous output $\operatorname{SNR}(\gamma)$ of MRC detector is given by [1]

$$
\gamma=\sum_{l=1}^{L} \gamma_{l}
$$

$L$ is the total number of independent and identically distributed (i.i.d.) branches used in diversity combiner. The instantaneous received SNR of the $l^{\text {th }}$ branch can be written as

$$
\gamma_{l}=X_{l}^{2}+Y_{l}^{2}, \quad l=1,2, \ldots, L .
$$

$X_{l}$ and $Y_{l}$ are independent and normally distributed random variables having zero mean and variance $\sigma_{x}^{2}$ and $\sigma_{y}^{2}$, respectively. The fading parameter is related to the variance as $q=\sigma_{y} / \sigma_{x}$ and $\sigma_{y}{ }^{2}=w\left[q^{2} /\left(1+q^{2}\right)\right]$. The closed-form expression for the conditional PDF of $\gamma$ is given by [15]

$$
\begin{aligned}
& p_{\gamma}(\gamma / w)=\left[-\left(\frac{\left(1+q^{2}\right) \gamma}{2 q^{2} w}\right)\right] \\
& \left(\frac{1+q^{2}}{2 q w}\right)^{L} \frac{\exp [(L)}{\Gamma(L)}{ }_{1} F_{1}\left(\frac{L}{2} ; L ; \frac{\left(1-q^{4}\right) \gamma}{2 q^{2} w}\right), \quad \gamma \geq 0
\end{aligned}
$$

where ${ }_{1} F_{1}(. ; . ;$.$) is the confluent hypergeometric function$ and $\Gamma(\cdot)$ is gamma function. Here, shadowing is modeled using Gamma distribution with PDF given by [2]

$$
p_{w}(w)=\frac{w^{(m-1)}}{\bar{\gamma}^{m} \Gamma(m)} \exp \left(-\frac{w}{\bar{\gamma}}\right), \quad m \geq 0
$$

where $\bar{\gamma}$ is average received SNR and $m$ is shaping parameter. Composite PDF of $\gamma$ can be easily evaluated by [1]

$$
p_{\gamma}(\gamma)=\int_{0}^{\infty} p_{\gamma}(\gamma / w) p_{w}(w) \mathrm{d} w .
$$

Substituting (4) and (5), into (6), we get

$$
\begin{aligned}
& p_{\gamma}(\gamma)= \int_{0}^{\infty}\left(\frac{1+q^{2}}{2 q w}\right)^{L} \frac{\exp \left[-\left(\frac{\left(1+q^{2}\right) \gamma}{2 q^{2} w}\right)\right] \gamma^{(L-1)}}{\Gamma(L)} . \\
&{ }_{1} F_{1}\left(\frac{L}{2} ; L ; \frac{\left(1-q^{4}\right) \gamma}{2 q^{2} w}\right) \frac{w^{(m-1)}}{\bar{\gamma}^{m} \Gamma(m)} \exp \left(-\frac{w}{\bar{\gamma}}\right) \mathrm{d} w
\end{aligned}
$$

where the hypergeometric function ${ }_{1} F_{1}(. ; . ;$.$) can be simpli-$ fied using [18, /9.210/1]. Transforming each exponential term by G-function using [19,/07.34.03.0228.01] and rearranging the terms, (7) can be simplified as

$$
\begin{aligned}
p_{\gamma}(\gamma) & =\sum_{n=0}^{\infty} \frac{s^{L} t^{n} \gamma^{(L+n-1)}\left(\frac{L}{2}\right)_{n}}{\Gamma(L+n) \Gamma(m) \bar{\gamma}^{m} n !} \cdot \\
& \int_{0}^{\infty} w^{(m-L-n)-1} G_{0,1}^{1,0}\left[\left.\frac{s \gamma}{q w}\right|_{0}\right] G_{0,1}^{1,0}\left[\frac{w}{\bar{\gamma}} \mid 0\right] \mathrm{d} w
\end{aligned}
$$

where $s=\left(1+q^{2}\right) / 2 q, t=\left(1-q^{4}\right) / 2 q^{2}$ and $\mathrm{G}(\cdot)$ is Meijer Gfunction, whereas $(x)_{n}$ denotes the Pochhammer's symbol. Using [18, /9.31/2] and [19,/07.34.21.0011.01] and after some mathematical manipulations, (8) can be written as

$$
\begin{aligned}
p_{\gamma}(\gamma) & =\sum_{n=0}^{\infty} \frac{t^{n}\left(\frac{L}{2}\right)_{n} q^{(n+L-m)} \gamma^{m-1}}{\Gamma(L+n) \Gamma(m) \bar{\gamma}^{m} n ! s^{n-m}} . \\
& G_{0,2}^{2,0}\left[\left.\frac{s \gamma}{q \bar{\gamma}}\right|_{0, n+L-m}\right] .
\end{aligned}
$$

By putting $q=1$ in the definition of $s$ and, $t$ equation (9) can be simplified to PDF of $K$ distribution.

\subsection{CDF}

Substituting (9) in the definitions of CDF, $P_{\gamma}(\gamma)=\int_{0}^{\gamma_{\text {th }}} p_{\gamma}(\gamma) \mathrm{d} \gamma$ and using [20] yields

$P_{\gamma}(\gamma)=$

$\sum_{n=0}^{\infty} \frac{t^{n}\left(\frac{L}{2}\right)_{n} q^{(n+L-m)}}{\Gamma(L+n) \Gamma(m) n ! s^{n-m}}\left(\frac{\gamma_{\mathrm{th}}}{\bar{\gamma}}\right)^{m} \cdot G_{1,3}^{2,1}\left[\frac{s \gamma_{\mathrm{th}}}{q \bar{\gamma}} \mid \begin{array}{c}1-m \\ 0, n+L-m,-m\end{array}\right]$. 
Outage probability is the probability that the instantaneous SNR falls below a predetermined threshold $\gamma_{\text {th }}$, and it can be easily obtained using (10).

\subsection{Moments}

The $i^{\text {th }}$ moment of $\gamma$ is defined by [1] as $E\left[\gamma^{i}\right]=\int_{0}^{\infty} \gamma^{i} p_{\gamma}(\gamma) \mathrm{d} \gamma$. With the aid of (9) and [19, /07.34.21.0009.01] it can be easily evaluated as

$$
\begin{aligned}
E\left[\gamma^{i}\right] & =\sum_{n=0}^{\infty} \frac{t^{n}\left(\frac{L}{2}\right)_{n} q^{(n+L+i)} \bar{\gamma}^{i}}{\Gamma(L+n) \Gamma(m) n ! s^{n+i}} . \\
& {[\Gamma(m+i)][\Gamma(n+L+i)] . }
\end{aligned}
$$

The AF is used to measure the severity of the channel and is defined as $A F=E\left[\gamma^{2}\right] / E[\gamma]^{2}-1$. AF of the $L-H G$ composite fading channel can be easily evaluated using (11).

\section{Channel Capacity}

Shannon's channel capacity gives the theoretical upper bound to the maximum rate of data transmission over a given channel with small error probability. It is an important performance parameter which is kept in mind while designing the system. Motivated by this fact, the closedform of channel capacity under optimal rate adaptation (ORA) and channel inversion fixed rate (CIFR) are presented here.

\subsection{ORA}

This scheme is more practical since the transmit power remains constant. The average capacity of a fading channel is a function of the bandwidth $B$ and $\gamma$. The channel capacity under this definition is expressed as [1]

$$
C_{\text {ora }}=B \int_{0}^{\infty} \log _{2}(1+\gamma) p_{\gamma}(\gamma) \mathrm{d} \gamma .
$$

Putting (9) in (12), and using [19, /07.34.03.0456.01], $[19, / 07.34 .21 .0011 .01]$, the above equation can be simplified as

$$
\begin{aligned}
& \frac{C_{\text {ora }}}{B}= \sum_{n=0}^{\infty} \frac{t^{n}\left(\frac{L}{2}\right)_{n} q^{(n+L-m)}}{\Gamma(L+n) \Gamma(m) \bar{\gamma}^{m} n ! s^{n-m}} . \\
& G_{2,4}^{4,1}\left[\frac{s}{q \bar{\gamma}} \mid \begin{array}{c}
-m, 1-m \\
0, n+L-m,-m,-m
\end{array}\right] .
\end{aligned}
$$

\subsection{CIFR}

Under this policy, transmitter allocates higher power to the channel with low SNR and lower power to higher SNR such that the constant received power is maintained. It is the capacity which gives lower bound of data rate through any channel. The channel capacity under CIFR scheme is defined as [1]

$$
C_{\text {cifr }}=B \log _{2}\left(1+\frac{1}{\int_{0}^{\infty} p_{\gamma}(\gamma) / \gamma \mathrm{d} \gamma}\right) .
$$

Using the result of (11) for $E[1 / \gamma]$ in (14), one can easily find out the final expression of $C_{\text {cifr. }}$.

\section{ASEP of Coherent Modulation Scheme}

In this section, we derive the closed-form expression of the coherent modulation scheme. The general formula for ASEP/ABEP is given by [1]

$$
P_{\mathrm{ave}}=E\left[P_{\mathrm{e}}(\gamma)\right]=\int_{0}^{\infty} P_{\mathrm{e}}(\gamma) p_{\gamma}(\gamma) \mathrm{d} \gamma
$$

where $P_{\mathrm{e}}(\gamma)$ is the instantaneous Symbol/Bit error rate of the modulation scheme and $p_{\gamma}(\gamma)$ is the PDF of the fading channel. The generalized probability of error for coherent modulation schemes is given by [21]

$$
P_{\mathrm{e}}(\gamma)=\sum_{d=1}^{D} \alpha_{d}\left[Q\left(\sqrt{c_{0} \gamma}\right)\right]^{d} .
$$

Here $D, \alpha_{d}$ and $c_{0}$ have their usual meaning as in [21]. It is analytically difficult to perform the averaging of instantaneous error probability in (15) by directly applying (16) in terms of Q-function. Thus, we need to resort to the approximate form of Q-function. There are various approximations to this Q-function suggested in the open literature [22-25]. However, all these approximations are not very precise as compared to second-order analytical approximation (as is evident from [23, Fig. 2 and 3]). The $Q(\cdot)$ function is approximated using [21], [23] as

$$
Q(\sqrt{t}) \approx \frac{a_{1}}{2} \exp \left(-b_{1} t / 2\right)+\frac{a_{2}}{2} \exp \left(-b_{1} t\right) .
$$

Here $a_{1}=0.3070, a_{2}=0.4389$ and $b_{1}=1.0510$. ASEP can be found by substituting (9) and (16) into (15) and using the approximate form of Q-function as in (17). The resultant expression is given by

$$
\begin{aligned}
P_{\mathrm{ave}} \approx & \sum_{n=0}^{\infty} \frac{t^{n}\left(\frac{L}{2}\right)_{n} q^{(n+L-m)}}{\Gamma(L+n) \Gamma(m) \bar{\gamma}^{m} n ! s^{n-m}} . \\
& \int_{0}^{\infty} \gamma^{m-1} G_{0,2}^{2,0}\left[\left.\frac{s \gamma}{q \bar{\gamma}}\right|_{0, n+L-m}\right] . \\
& \sum_{d=1}^{D} \alpha_{d}\left[\frac{a_{1}}{2} \exp \left(-\frac{b_{1} c_{0} \gamma}{2}\right)+\frac{a_{2}}{2} \exp \left(-b_{1} c_{0} \gamma\right)\right]^{d} \mathrm{~d} \gamma .
\end{aligned}
$$

This integral can be solved by expanding the square bracket of the integrand using Binomial expansion 
$(x+y)^{n}=\sum_{k=0}^{n} C(n, k) x^{n-k} y^{k}$. Transforming each exponential term by G-function using [19, /07.34.03.0228.01] and then using the results of integral of the product of two G-functions in [19, /07.34.21.0011.01], generalized closedform solution of ASEP for all formats of coherent modulation techniques is given by

$$
\begin{aligned}
P_{\mathrm{ave}} & \approx \sum_{n=0}^{\infty} \frac{t^{n}\left(\frac{L}{2}\right)_{n} q^{(n+L)}}{\Gamma(L+n) \Gamma(m) n ! s^{n}} \sum_{d=1}^{D} \alpha_{d} . \\
& \sum_{r=0}^{d} C(d, r)\left(\frac{a_{1}}{2}\right)^{d-r}\left(\frac{a_{2}}{2}\right)^{r} \cdot \\
& G_{2,1}^{1,2}\left[\frac{(d+r) b_{1} c_{0} q \bar{\gamma}}{2 s} \mid \begin{array}{c}
1-m, 1-n-L \\
0
\end{array}\right]
\end{aligned}
$$

where $C(d, r)$ is the combination of $r$ objects from a set of $d$ objects. The next section deals with the derivation of the average probability of error for non-coherent modulation techniques.

\section{ASEP/ABEP of Non-Coherent Modulation Scheme}

There are certain situations in wireless communication where phase recovery at the receiver cannot be tracked accurately. In such scenarios, the wireless communication system must depend on the non-coherent reception, such as square-law detection of Frequency-shift-keying (FSK) signal [22] or differential coherent modulation technique such as Differential phase-shift-keying (DPSK). Some of the related works in evaluating the closed-form expressions of the non-coherent techniques has been carried out in the past [23], [26], [27]. In this section, we have proposed the analytical formulas for non-coherent schemes over composite HG fading with diversity.

\subsection{Multiple-FSK (MFSK)}

The instantaneous symbol error probability of MFSK is given by [22]

$$
P_{\mathrm{e}}(\gamma)=\sum_{p=1}^{M-1} C(M-1, p) \frac{(-1)^{p+1}}{p+1} \exp \left(-\frac{p}{p+1} \gamma\right) .
$$

Here, each symbol of MFSK represents $\left(\log _{2} M\right)$ bits. The ASEP expression for MFSK is obtained by substituting (20) and (9) into (15) and using [19, /07.34.03.0228.01], $[19, / 07.34 .21 .0011 .01]$

$$
\begin{aligned}
& P_{\text {ave }}=\sum_{n=0}^{\infty} \frac{t^{n}\left(\frac{L}{2}\right)_{n} q^{(n+L)}}{\Gamma(L+n) \Gamma(m) n ! s^{n}} \sum_{p=1}^{M-1} C(M-1, p) \frac{(-1)^{p+1}}{p+1} . \\
& G_{2,1}^{1,2}\left[\frac{p q \bar{\gamma}}{(p+1) s} \mid \begin{array}{c}
1-m, 1-n-L \\
0
\end{array}\right] .
\end{aligned}
$$

\subsection{Differential-QPSK (DQPSK)}

Bit error probability for non-coherent DQPSK is given by [22]

$$
P_{\mathrm{e}}(\gamma)=Q_{1}(a \sqrt{\gamma}, b \sqrt{\gamma})-\frac{1}{2} \mathrm{I}_{0}(a b \gamma) \exp \left(-\frac{a^{2}+b^{2}}{2} \gamma\right)
$$

where $Q_{1}(a \sqrt{\gamma}, b \sqrt{\gamma})$ is the Marcum Q-function of the first order and $a=\sqrt{2(1-1 / \sqrt{2})}, b=\sqrt{2(1+1 / \sqrt{2})}$. Substituting (22) and (9) into (15) and using the expressions of Marcum Q-function and modified Bessel function [22], one can write the resulting expression as

$$
\begin{aligned}
P_{\mathrm{ave}} & =\int_{0}^{\infty} \sum_{n=0}^{\infty} \frac{t^{n}\left(\frac{L}{2}\right) q_{n}^{(n+L-m)} \gamma^{m-1}}{\Gamma(L+n) \Gamma(m) \bar{\gamma}^{m} n ! s^{n-m}} . \\
& G_{0,2}^{2,0}\left[\left.\frac{s \gamma}{q \bar{\gamma}}\right|_{0, n+L-m]}\right] \\
& \left\{\begin{array}{l}
\exp (-x \gamma) \sum_{k=0}^{\infty}\left(\frac{a}{b}\right)^{k} \sum_{l=0}^{\infty} \frac{(r \gamma)^{z}}{l ! \Gamma(l+k+1)} \\
-\frac{1}{2} \exp (-x \gamma) \sum_{p=0}^{\infty} \frac{(r \gamma)^{2 p}}{(p !)^{2}}
\end{array}\right\} \mathrm{d} \gamma
\end{aligned}
$$

where $x=\left(a^{2}+b^{2}\right) / 2, r=a b / 2$, and $z=2 l+k$. Using [19, /07.34.03.0228.01] and [19,/ 07.34.21.0011.01], we can obtain the final result for ABEP of DQPSK as

$$
\left.\begin{array}{rl}
P_{\mathrm{ave}} & =\sum_{n=0}^{\infty} \frac{t^{n}\left(\frac{L}{2}\right)_{n} q^{(n+L)}}{\Gamma(L+n) \Gamma(m) n ! s^{n}} . \\
\sum_{k=0}^{\infty}\left(\frac{a}{b}\right)^{k} \sum_{l=0}^{\infty} \frac{(r)^{z}}{l ! \Gamma(l+k+1)}\left(\frac{q \bar{\gamma}}{s}\right)^{z} \\
\cdot G_{2,1}^{1,2}\left[\frac{x q \bar{\gamma}}{s} \mid 1-(z+m), 1-(z+n+L)\right] \\
-\frac{1}{2} \sum_{p=0}^{\infty} \frac{(r)^{2 p}}{(p !)^{2}}\left(\frac{q \bar{\gamma}}{s}\right)^{2 p} \cdot \\
\cdot G_{2,1}^{1,2}\left[\frac{x q \bar{\gamma}}{s} \mid 1-(2 h+m), 1-(2 h+n+L)\right] \\
0
\end{array}\right\} .
$$

\section{Numerical Results and Discussion}

In this section, we confirm the validity of our performance parameters. All the computations and simulations were carried out in MATLAB (version R2014a). The default value of $\bar{\gamma}$ is taken as unity in all the calculations. To validate the accuracy of the derived expressions, MonteCarlo simulations are also included with $10^{7}$ numbers of samples for generating $L-\mathrm{HG}$ composite distribution. It can be observed that the simulation results are in close match with the numerical results obtained by keeping enough number of terms in the infinite series. In Fig. 1, outage probability is plotted against the normalized outage thresh- 
old $\left(\gamma_{\text {th }} / \bar{\gamma}\right)$ for several values of $m$ and $q$ using closed-form expression (10). It is evident from the results that outage probability increases with the increase in $\gamma_{\text {th }}$ indicating an increase in the likelihood of failure to achieve a given threshold level.

As expected, the outage probability is shown to decrease with increase in $m$ and $q$, hinting an improvement in performance of the receiver. The effect of channel condition improvement with diversity is clearly shown in the plot. AF is plotted as a function of $m$ for several values of $q$ in Fig. 2. It can be easily observed that AF reduces as $m$ and $L$ increases, showing improved performance. Furthermore, as $q$ increases, AF plot shifts downwards, while it can also be observed that the gap among the curves decreases as $L$ increases. Moreover, in Fig. 3 the impact of the fading parameter $q$ on the channel capacity is illustrated for various values of diversity order $L$ based on (13). The graph reveals that increasing the values of $q$ or $L$ both helps overcome the effects of fading. Figure 4 depicts the average channel capacity as a function of the average received SNR for the ORA and CIFR schemes with different values of the fading parameter $q$. The outputs of Monte-Carlo

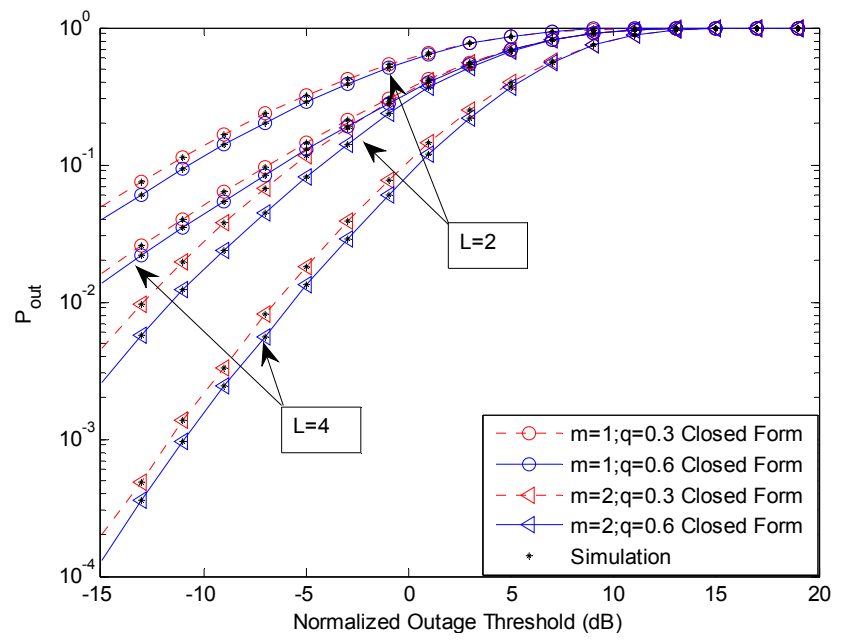

Fig. 1. Diversity effect on outage probability for several values of $m$ and $q$.

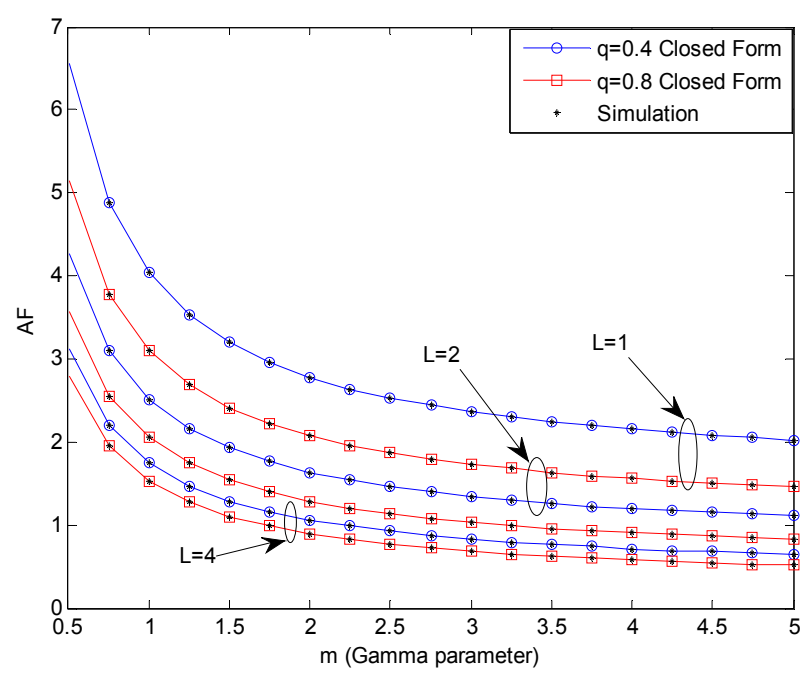

Fig. 2. AF versus $m$ for several values of $q$ and $L$.

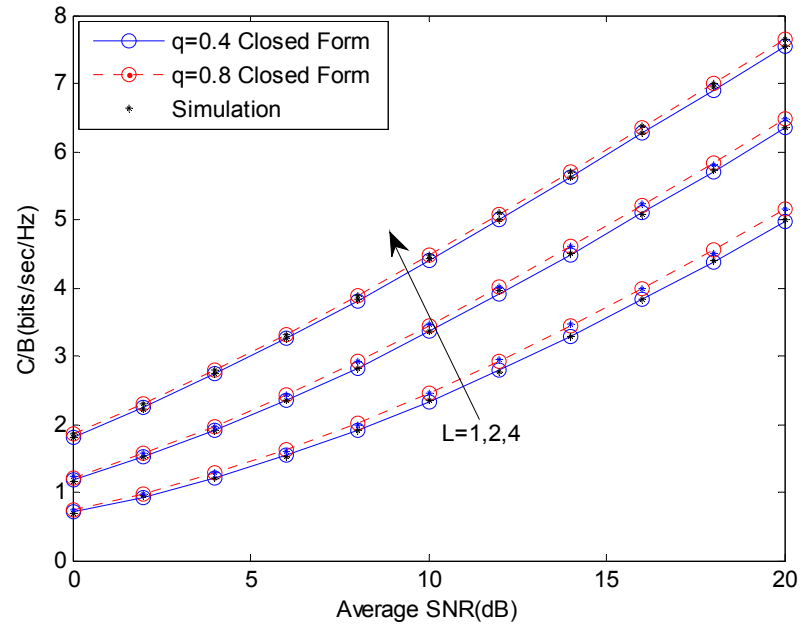

Fig. 3. Effect of diversity and fading parameter on channel capacity with ORA for $m=1$.

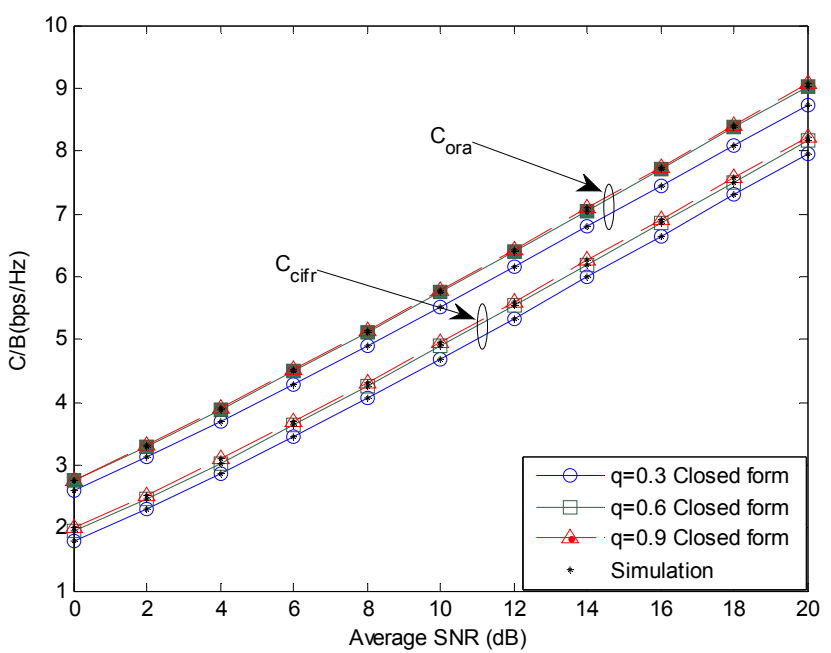

Fig. 4. Channel capacity with ORA and CIFR as a function of average SNR for $m=2$ and $L=4$.

simulations are also provided and shown to exactly match with the analytical results. It is evident that, as the average received SNR increases, the capacity of both the transmission schemes improves. We also find that CIFR achieves the lower capacity than ORA. This is because of the fact that CIFR uses a fixed transmission data rate, whereas a large amount of transmitting power is required to compensate for the deep channel fades.

Comparison of QPSK and DQPSK (non-coherent) schemes for different values of $L$ is shown in Fig. 5. As observed, an excellent match between the analytical results produced by putting closed-form expression (19) for QPSK and (24) for DQPSK with the simulations results are achieved. It is observed that the performance of the system improves with an increase in $L$ as shown by downshifting of the plots. Secondly, QPSK outperforms DQPSK significantly. In Fig. 6, ASEP of DEQPSK has been plotted over the composite fading channel for several values of $m, q$ and $L$. The theoretical curves have been constructed based on expression (19). It is observed that the plots shift downwards with the increase in the fading and shape parameters indicating an improvement in the system performance. 
Figure 7 demonstrates the plots for $M$-ary QAM using the analytical expression (19) over $L$-HG composite fading channel under the various constellation size $M=2,4$ and 8 . It is noted from the results that the plot shifts upwards for the higher constellation.

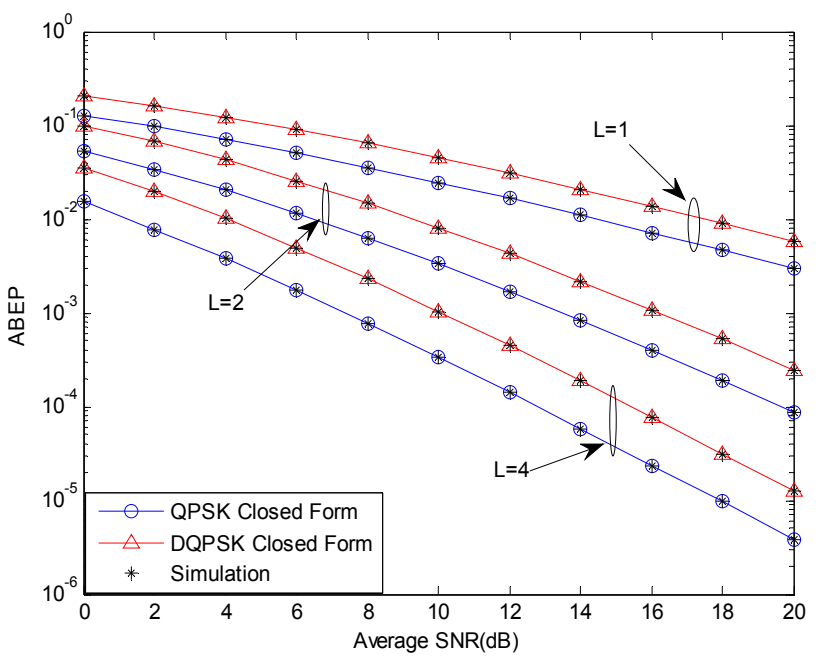

Fig. 5. ABEP Comparison between QPSK (coherent) and DQPSK (non-coherent) for different values of $L$.

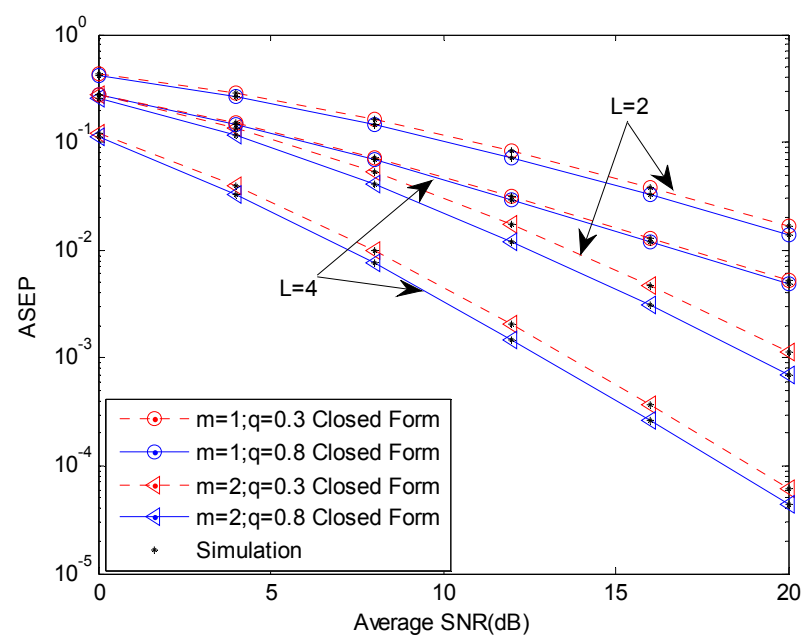

Fig. 6. ASEP of DEQPSK for HG for several values of $m, q$ and $L$.

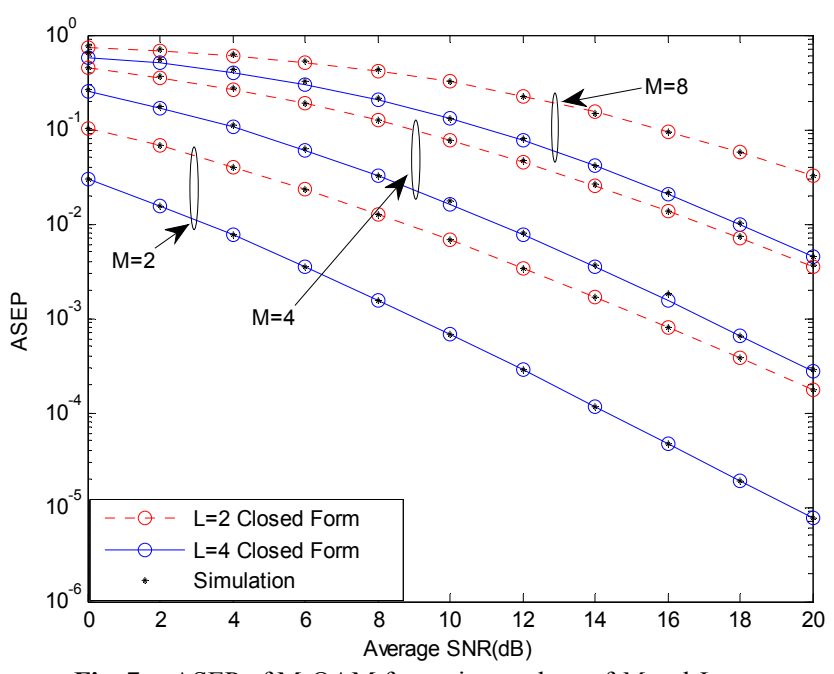

Fig. 7. ASEP of M-QAM for various values of $M$ and $L$.

\begin{tabular}{|c|c|c|c|c|}
\hline \multirow{2}{*}{ Modulation } & \multicolumn{2}{|c|}{$L=1$} & \multicolumn{2}{c|}{$L=4$} \\
\cline { 2 - 5 } & $q=0.4$ & $q=0.8$ & $q=0.4$ & $q=0.8$ \\
\hline BPSK (19) & 14 & 2 & 26 & 4 \\
\hline QPSK (19) & 16 & 3 & 29 & 5 \\
\hline 8-PSK (19) & 20 & 4 & 35 & 6 \\
\hline 2-PAM (19) & 11 & 2 & 21 & 3 \\
\hline 8-PAM (19) & 22 & 4 & 39 & 6 \\
\hline 2-QAM (19) & 14 & 2 & 27 & 4 \\
\hline 8-QAM (19) & 21 & 4 & 37 & 6 \\
\hline DEQPSK 19) & 17 & 3 & 31 & 5 \\
\hline BFSK (21) & 15 & 3 & 29 & 5 \\
\hline DQPSK (24) & 13 & 2 & 27 & 4 \\
\hline
\end{tabular}

Tab. 1. Number of terms $(N)$ required for accuracy at 7 th place of the decimal digit in the numerical evaluation of (19), (21) and (24).

ABEP/ASEP expressions presented herein are given in terms of infinite series. We have truncated the series by including the finite number of terms $N$ ensuring to achieve accuracy at the seventh place of the decimal digit. In Tab. 1, we have calculated the number of terms required to achieve this accuracy in (19), (21) and (24) as a function of $q$ and $L$ with $m=1$. The number of terms needed to be summed to achieve the desired accuracy depends on $q, L$ and the modulation technique being used. The value of $N$ decreases with increase in $q$, indicating that more number of terms are required for more severe fading conditions. It can be observed that we need more number of terms for higher diversity order system.

\section{Conclusions}

Taking note of the fact that Hoyt is known to capture the severe multipath fading and Gamma distribution is most widely used to model shadowing, we have analyzed the performance of $L$-HG composite fading model. Closedform expressions for PDF of instantaneous SNR, outage probability, AF, channel capacity and ASEP/ABEP of the composite $L-H G$ were obtained in terms of generalized hypergeometric functions and the corresponding results are demonstrated. These expressions can be useful in the performance evaluation of $L-H G$ composite fading environment. Moreover, the analytical results are found to be in close agreement with the simulation results.

\section{Acknowledgments}

The authors would like to thank Vidhi Rana and Pushpraj Chauhan and the anonymous reviewers for their useful suggestions for improving the presentation of the material in this paper.

\section{References}

[1] SIMON, M. K., ALOUINI, M. S. Digital Communication over Fading Channels. 2nd ed. New York: John Wiley \& Sons, 2004. ISBN: 0-471-64953-8 
[2] SHANKAR, P. Fading and Shadowing in Wireless Systems. New York: Springer, 2012. ISBN: 978-1-4614-0366-1

[3] YOUSSEF, N., ELBAHRI, W., PATZOLD, M., ELASMI, S. On the crossing statistics of phase processes and random FM noise in Nakagami-q mobile fading channels. IEEE Transactions on Wireless Communications, 2005, vol. 4, no. 1, p. 24-29. DOI: $10.1109 /$ TWC.2004.840251

[4] NAKAGAMI, M. The m-distribution-A general formula of intensity distribution of rapid fading. In Hoffman, W. G., Ed. Statistical Methods in Radio Wave Propagation. Pergamon Press, 1960, p. 3-36. DOI: 10.1016/B978-0-08-009306-2.50005-4

[5] PARIS, J. Nakagami-q (Hoyt) distributions function with applications. Electronics Letters, 2009, vol. 45, no. 4, p. 749-751. DOI: 10.1049/el:20093427

[6] PARIS, J., JIMENEZ, D. Outage probability analysis for Nakagami-q (Hoyt) fading channels under Rayleigh interference. IEEE Transactions on Wireless Communications, 2010, vol. 9, no. 4 , p. 1272-1276. DOI: 10.1109/TWC.2010.04.090295

[7] RADAYDEH, R. Performance of cellular mobile systems employing SNR-based GSC in the presence of Rayleigh and Nakagami-q co-channel interferers. IEEE Transactions on Vehicular Technology, 2009, vol. 58, no. 6, p. 3081-3088. DOI: $10.1109 /$ TVT.2008.2011276

[8] SHANKAR, P. M. A Nakagami-N-gamma model for shadowed fading channels. Wireless Personal Communications, 2012, vol. 64, no. 5, p. 665-680. DOI: 10.1007/s11277-010-0211-5

[9] SHANKAR, P. M. An overview of shadowed fading wireless channels in terms of a cascaded approach. Physical Communication, June 2015, vol. 15, no. C, p. 59-65. DOI: 10.1016/j.phycom.2015.02.001

[10] SINGH, R., SONI, S. K., RAW, R. S., KUMAR, S. A new approximate closed-form distribution and performance analysis of a composite Weibull/log-normal fading channel. Wireless Personal Communications, 2017, vol. 92, no. 3, p. 883-900. DOI: $10.1007 / \mathrm{s} 11277-016-3583-3$

[11] ABDI, A., KAVEH, M. K distribution: an appropriate substitute for Rayleigh-lognormal distribution in fading-shadowing wireless channels. Electronics Letters, 1998, vol. 34, no. 9, p. 851-852. DOI: 10.1049/el:19980625

[12] BITHAS, P., SAGIAS, N., MATHIOPOULOS, P., KARAGIANNIDIS, G., RONTOGIANNIS, A. On the performance analysis of digital communications over generalized$\mathrm{K}$ fading channels. IEEE Communications Letters, 2006, vol. 4, no. 10 , p. 353-355. DOI: 10.1109/LCOMM.2006.1633320

[13] BITHAS, P. Weibull-Gamma composite distribution: alternative multipath/shadowing fading model. Electronics Letters, 2009, vol. 45 , no. 14 , p. 749-751. DOI: $10.1049 /$ el.2009.0534

[14] ZHANG, J., MATTHAIOU, M., TAN, Z., WANG, H., KAVEH, M. Performance analysis of digital communication systems over composite $\eta-\mu$ /gamma fading channels. IEEE Transactions on Vehicular Technology, 2012, vol. 61, no. 7, p. 3114-3124. DOI: 10.1109/TVT.2012.2199344

[15] STEFANOVIC, C., JAKSIC, B., SPALEVIC, P., PANIC, S., TRAJCEVSKI, Z. Performance analysis of selection combining over correlated Nakagami-m fading channels with constant correlation model for desired signal and cochannel interference. Radioengineering, 2013, vol. 22, no. 4, p. 1176-1181.

[16] JAKSIC, B., STEFANOVIC, D., STEFANOVIC, M., SPALEVIC, P., MILENKOVIC, V. Level crossing rate of macrodiversity system in the presence of multipath fading and shadowing. Radioengineering, 2015, vol. 24, no. 1, p. 185-191. DOI: $10.13164 /$ re.2015.0185

[17] SUBADAR, R., SAHU, P. R. Performance of L-MRC receiver over independent Hoyt fading channels. In Proceedings of the
National Conference on Communications. Chennai (India), 2010, p. 1-5. DOI: $10.1109 /$ NCC.2010.5430232

[18] GRADSHTEYN, S., RYZHIK, I. Table of Integrals, Series, and Products. $6^{\text {th }}$ ed. New York: Academic Press 2000. ISBN: 0-122-94757-6

[19] WOLFRAM. Wolfram function site. [Online] Cited 2017-01. Available at: http://functions.wolfram.com/PDF/MeijerG.pdf

[20] ADAMCHIK, V., MARICHEV, O. The algorithm for calculating integrals of hypergeometric type functions and its realization in REDUCE system. In Proceedings of International Conference on Symbolic and Algebraic Computation. New York (USA), 1990, p. 212-224. DOI: $10.1145 / 96877.96930$

[21] KHANDELWAL, V., KARMESHU. A new approximation for average symbol error probability over log-normal channels. IEEE Wireless Communications Letters, 2014, vol. 3, no. 1, p. 58-61. DOI: 10.1109/WCL.2013.111113.130525

[22] PROAKIS, J. G., SALEHI, M. Digital Communications. $5^{\text {th }}$ ed. New York: McGraw-Hill, 2008. ISBN: 978-0-07-295716-7

[23] OLABIYI, O., ANNAMALAI, A. Invertible exponential-type approximations for the Gaussian probability integral $\mathrm{Q}(\mathrm{x})$ with applications. IEEE Wireless Communications Letters, 2012, vol. 1, no. 5, p. 544-547. DOI: 10.1109/WCL.2012.080112.120232

[24] PADMAPRIYA, S., TAMILARASI, M. Co-channel interference avoidance algorithm for closed access femtocell networks. IETE Technical Review, 2015, vol. 33, no. 3, p. 280-296. DOI: $10.1080 / 02564602.2015 .1088413$

[25] LAOURINE, A., STEPHENNE, A., AFFES, S. Estimating the ergodic capacity of log-normal channels. IEEE Communications Letters, 2007, vol. 11, no. 7, p. 568-570. DOI: 10.1109/LCOMM.2007.070302

[26] HELIOT, F., GHAVAMI, M., NAKHAI, M. R. An accurate closed-form approximation of the average probability of error over log-normal fading channel. IEEE Transactions on Wireless Communications, 2008, vol. 7, no. 5, p. 1495-1500. DOI: 10.1109/TWC.2008.061019

[27] SIMON, M. K., ALOUINI, M. S. A unified approach to the probability of error for non-coherent and differentially coherent modulations over generalized fading channels. IEEE Transactions on Communications, 1996, vol. 46, no. 12, p. 1625-1638. DOI: $10.1109 / 26.737401$

\section{About the Authors...}

Sandeep KUMAR was born in Delhi, India in 1983. He received his Bachelor of Technology in Electronics and Communication from Kurukshetra University, India in 2004 and Master of Engineering in Electronics and Communication from Thapar University, Patiala, India in 2007. $\mathrm{He}$ is currently pursuing Ph.D. from Delhi Technological University, India. Presently he is working as a Member (Research Staff) at the Central Research Laboratory, Ghaziabad, Bharat Electronics Limited. His research interest includes the study of wireless channels, performance modeling of fading channels and radio network planning.

Sanjay Kumar SONI was born in UP, India in 1975. He received his Bachelor of Engineering in Electronics Engineering from Madan Mohan Malviya Engineering College, Gorakhpur in 1997, M. Tech. degree in Communication Engineering from IIT Kanpur in 2004, and Ph.D. in Wireless Communication Engineering from IIT Kharagpur in 
2011. Currently, he is an Associate Professor in the Department of Electronics and Communication in G. B. Pant Engineering College, Pauri (Uttrakhand). He has more than 14 years teaching experience. His research interest includes propagation modeling and characterization of wireless channel, time-domain analysis of propagation channel for UWB signals.

Priyanka JAIN received B.E. degree in Electronics \& Telecommunication, M.Tech. in Microwave Engineering from Delhi University, and Ph.D. from Guru Gobind Singh Indraprastha University, Delhi. She has over 9 years of teaching experience at Indira Gandhi Institute of Technology, GGSIP University. At present, she is an Assistant Professor in ECE department, Delhi Technological University. She has published a large number of papers in national and international journal in the field of digital signal processing. Her teaching and research are in the area of signal processing, analog electronics and microwave. 